$\xi=\mathbf{\alpha}$

\title{
Preparation and characterization of polyaniline/polyvinyl alcohol nanocomposite by laser irradiation
}

\author{
N A M Shahin ${ }^{1}$, S Abd El Mongy. ${ }^{1}$, R Kamal ${ }^{1}$, A B El- Bially ${ }^{1}$, A A Shabaka ${ }^{2}$, F Barakat ${ }^{1}$ \\ ${ }^{1}$ Department of Physics, Faculty of Women for Arts, Science and Education, Ain Shams University, Egypt \\ ${ }^{2}$ Department of Spectroscopy, Physics Division, National Research Center, Giza, 12622, Egypt \\ E-mail: samiraabd_32@yahoo.com
}

\begin{abstract}
Polyaniline (PANI) - Polyvinyl alcohol (PVA) nanocomposite were prepared using laser irradiation method. X-ray diffraction results showed that, (PANI/PVA) nanocomposite exhibited amorphous nature of polymer. The electronic transition will be studied using Ultraviolet-Visible spectrometer (UV-Vis). The real part of dielectric constant ( $(\dot{)})$ and imaginary part $\left(\varepsilon^{\prime \prime}\right)$ were studied. Also, the relaxation time was calculated.
\end{abstract}

Keywords: PANI/PVA Nanocomposites; XRD and UV-Visible; Dielectric Constant

\section{Introduction}

Polyaniline (PANI) is one of the most extensively studied conductive polymers. Potential applications include uses in rechargeable batteries, sensors, switchable membranes, anticorrosive coatings, and electronic devices [1]. Polyaniline is commercially attractive owing to its easy synthesis either through chemical or electrochemical methods, good environmental stability, ease of conductivity control, and inexpensive production in large quantities. However, like other conductive polymers, polyaniline by itself cannot be easily fabricated as a thin-film form with good mechanical properties. Thus, its practical use has been limited [2].

Nanocomposites are a special class of materials originating from suitable combinations of two or more such nanoparticles or nanosized objects in some suitable technique, resulting in materials having unique physical properties and wide application potential in diverse areas that can be formed into a useful object which can be subsequently used [3], [4]. 4]. Novel properties of nanocomposites [5], [6] can be derived from the successful combination of the individual characteristics of parent constituents into a single material. To exploit the full potential of the technological applications of the nanomaterials, it is thus extremely important to endow them with good processability.

In the present work, Polyaniline/polyvinylalcohol nanocomposite were Prepared. The particle size was calculated from X-ray diffraction (XRD). The ultraviolet-visible (UV-Vis.) spectra were discussed. Also, the dielectric constants were studied.

\section{Experimental details}

Aniline hydrochloride $\left(\mathrm{C}_{6} \mathrm{H}_{8} \mathrm{CIN}\right)$ (AniHCl, molecular weight $(\mathrm{Mw})=129.59 \mathrm{~g} / \mathrm{mol}) 59 \mathrm{~g} / \mathrm{mol}$ were obtained from Oxford and Polyvinyl-Alcohol $\left(\mathrm{C}_{6} \mathrm{H}_{7} \mathrm{~N}\right)$ of $\mathrm{MW}=6000 \mathrm{~g} / \mathrm{mol}$ with minimum assay $99.5 \%$ purchased from Mallinckrott.

The PVA solution was first prepared by dissolving PVA powder (5 g) in $100 \mathrm{ml}$ deionized water and stirring for 3 hours. AniHCl powder (1.5 g) was added into the PVA solution at room temperature and continuously stirred for 3 hours until a clear solution was obtained. The solution was paused in Petri dish and left to dry in air at room temperature to obtain the desired films. The $\mathrm{PVA} / \mathrm{AniHCl}$ blended films were cut into several pieces to facilitate to be exposed to laser irradiation using (CW-Diode -807 at power $1500 \mathrm{~mW})$ at different irradiation time at $(3,6,9,12$ and 15) mints. UV- Visible measurements were performed on all samples, using a V-570 UV/Vis/ NIR spectrometer in absorption mode in the wavelength range of 190-2500 $\mathrm{nm}$. X-ray diffraction measurements was obtained using an X-ray diffractometer Bruker AXS in the $2 \theta$ Range $20-80^{\circ}$ and dielectric constants were determined using LCR bridge mode 3531 Hioki.

\section{Result and discussion}

\subsection{X-Ray diffraction}

The X-Ray diffraction pattern of the PANI/PVA nanocomposite prepared at different irradiation times $(3,6,9,12$, and 15) mints is shown in Fig. (1). From Fig. (1), we observed that, no sharp peaks which confirm the amorphous nature of polymer blends [7]. The particle size $\mathrm{D}$ was determined from the XRD pattern according to the Scherrer's equation [8], [9].

$\mathrm{D}=\frac{\mathrm{K} \lambda}{\beta \cos \theta}$

Where, $\mathrm{D}$ is the particle size, $\mathrm{K}$ is the shape factor for the Particle $(\sim 0.9), \beta$ is the full width at half maximum of the peak in radian, and $\theta$ is the angle obtained from $2 \theta$ value corresponding to maximum intensity peak in XRD pattern.

The particle size are decrease with increasing the irradiation time as shown in Table (1). The decrease in the particle size with increasing irradiation times may be due to hydrogen bonding between the imine and amine nitrogen sites. 
Table 1: The Particle Size of PANI/PVA Nanocomposite at Different Irradiation Time (3, 6, 9, 12 and 15) Mints

\begin{tabular}{ll}
\hline Time $(\mathrm{min})$ & Particle Size $(\mathrm{nm})$ \\
\hline 3 & 9.9 \\
6 & 7.8 \\
9 & 6.2 \\
12 & 5.14 \\
15 & 4.94 \\
\hline
\end{tabular}

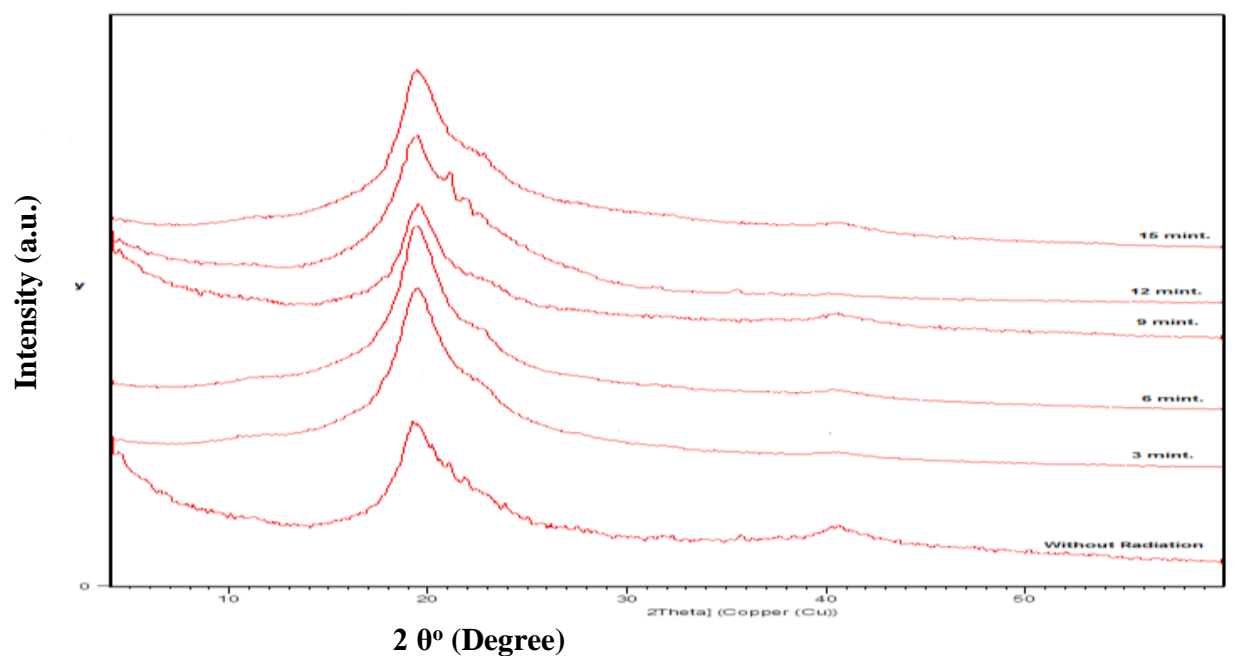

Fig. 1: X-Ray Diffraction Pattern of PANI/PVA Nanocomposite at Different Irradiation Times (3, 6,9,12 and 15) Min.

\subsection{UV-Vis absorption spectroscopy}

The UV-Vis spectra of PANI/PVA composite prepared before and after exposure to irradiation is shown in Figure (2). By comparing PANI/PVA composite and PANI/PVA composite at different irradiation times $(3,6,9,12$ and 15) min., it can be observed that, with increasing irradiation time, the $\pi-\pi^{*}$ absorption band at 230 $\mathrm{nm}$ and $\mathrm{n}-\pi^{*}$ absorption band at $286 \mathrm{~nm}$ are slightly shifted towards higher wavelength at $(232,238,236,236,236) \mathrm{nm}$ and $(292,296.296,294,296) \mathrm{nm}$ respectively. This red shift which indicates that, the conjugation chain of PANI enlarged and expected to lower the absorption energy [10-12]. Also, there are new bands appeared at $(556,564,564,562$ and 564) $\mathrm{nm}$ for irradiation time at $(3,6,9,12$ and 15) min. respectively which corresponded to $\pi$ - polaron transition, which are characteristic the protonated PANI [13-16]. This band is ascribed to cation radicals [17], and indicates the formation of bipolarons and PANI in emeraldine salt form [18-20].

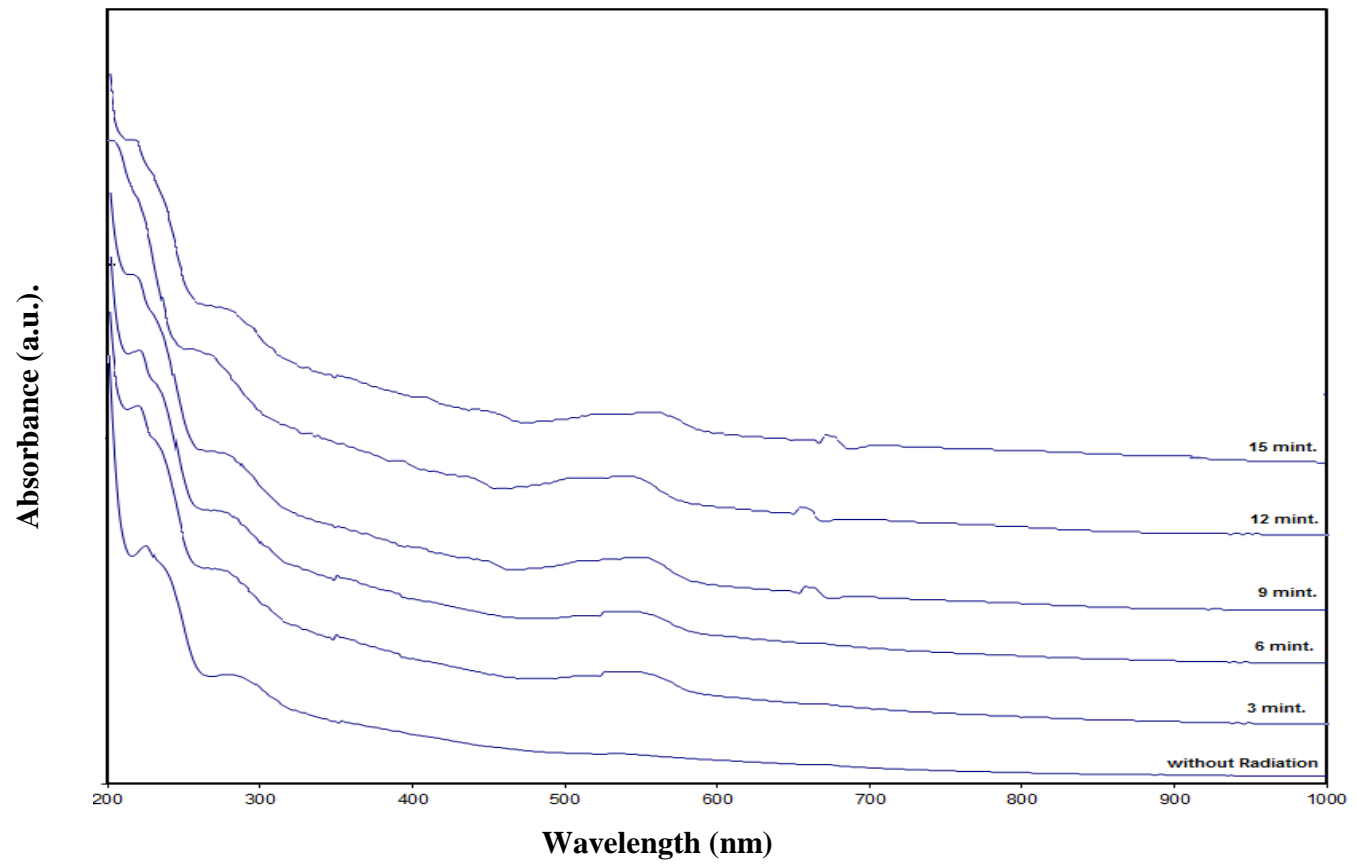

Fig. 2: UV-Visible Spectrum of PANI/PVA Nanocomposite before and After Irradiation at Different Times (3, 6, 9, 12 and 15) Min. 


\subsection{Dielectric properties}

\subsubsection{Dielectric constant}

The variation of the real part of dielectric constant PANI/PVA composite and PANI/PVA nanocomposite films prepared at different irradiation times, (3,6,9,12 and 15 )min., as a function of frequency ranging from $500 \mathrm{kHz}$ to $5 \mathrm{MHz}$ and measured at different temperatures $(303,333,363$ and 393$) \mathrm{K}^{\circ}$ are shown in Figure (3(a-f)). From figure, it is clear that the dielectric constant increases with increasing of temperature. This increase may be attributed to the orientation of the dipoles which formed from the charge carriers. The increase in temperature increases the ability of dipoles to align along the applied field [21] and thereby increasing the effective length of dipole which, in turn, should cause increasing the capacitance [22] and hence increase the dielectric constant.

(A)

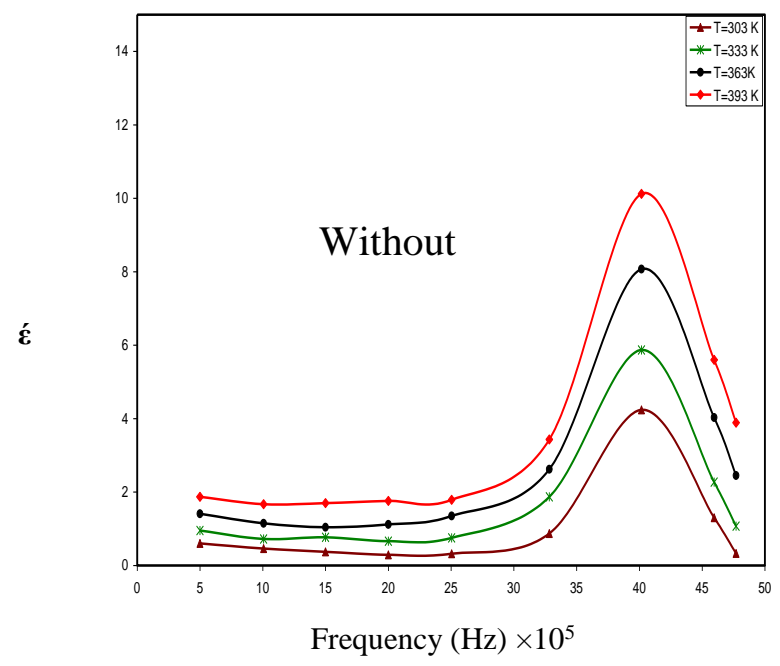

(C)

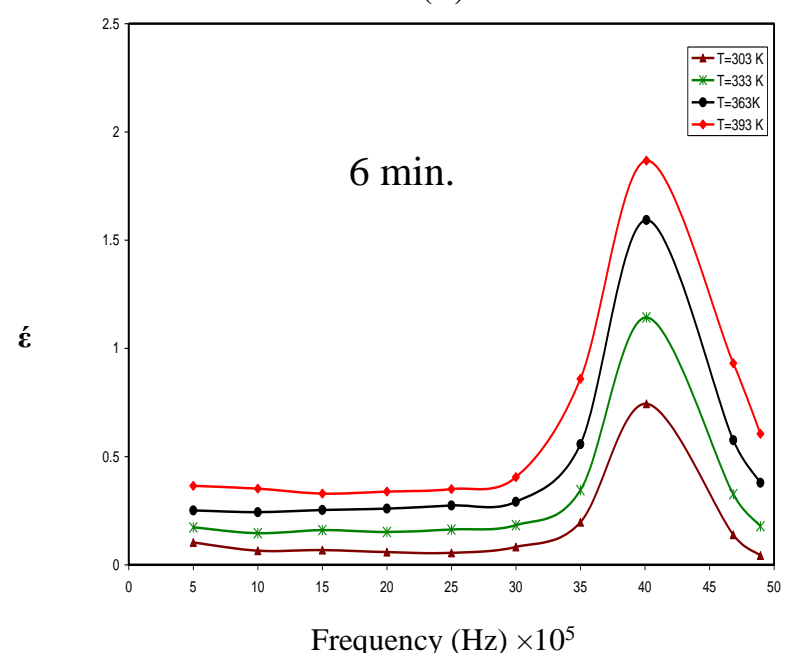

These phenomena in dielectric materials are associated with a frequency- dependent orientational polarization. However, in nanocrystalline materials, the space charge polarization plays a significant role. This is due to the large number of electron and hole traps at the surface of nanomaterials.

At low frequencies, the permanent dipoles align themselves along the field and contribute fully to the total polarization of the dielectric. At higher frequencies, the variation in the field is too rapid for the dipoles to align themselves, so their contribution to the polarization and, hence, to the dielectric permittivity can become negligible. Therefore, the dielectric permittivity decreases with increasing frequency. $\dot{\varepsilon}$

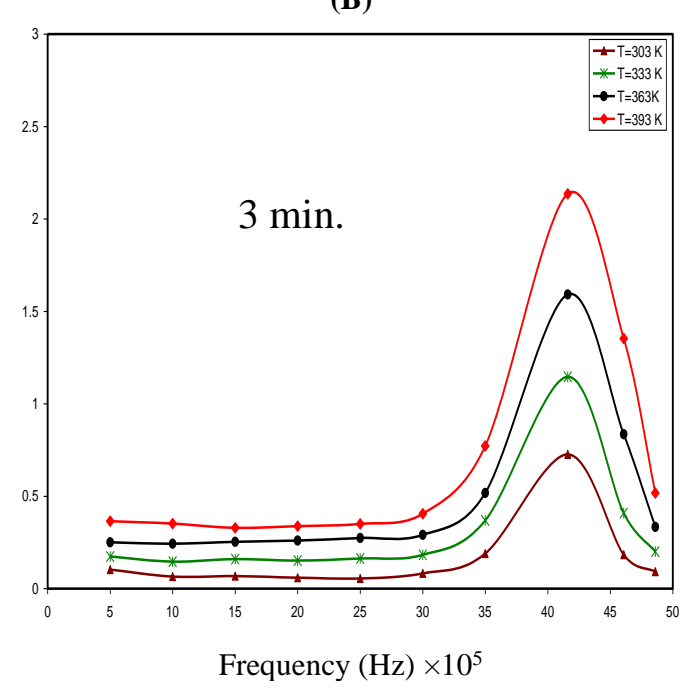

(D)

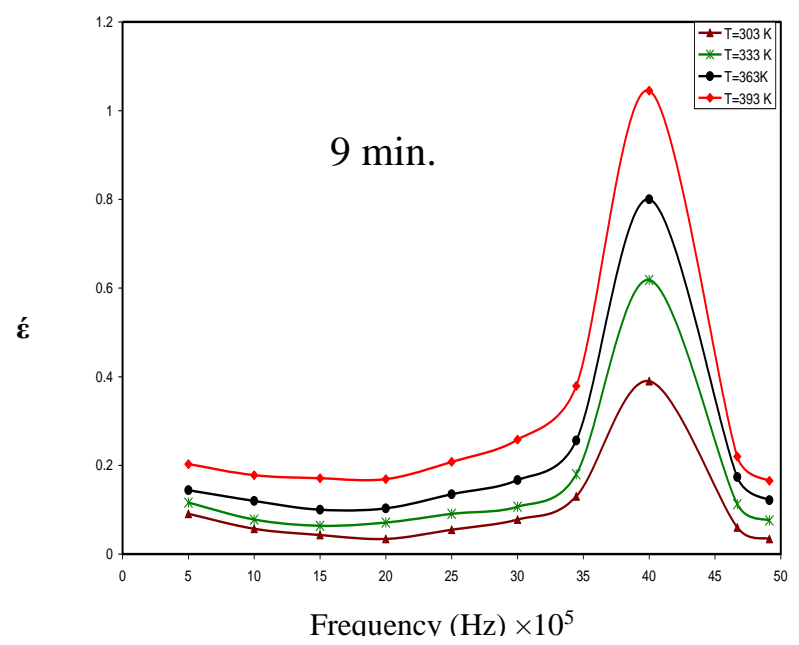


(E)

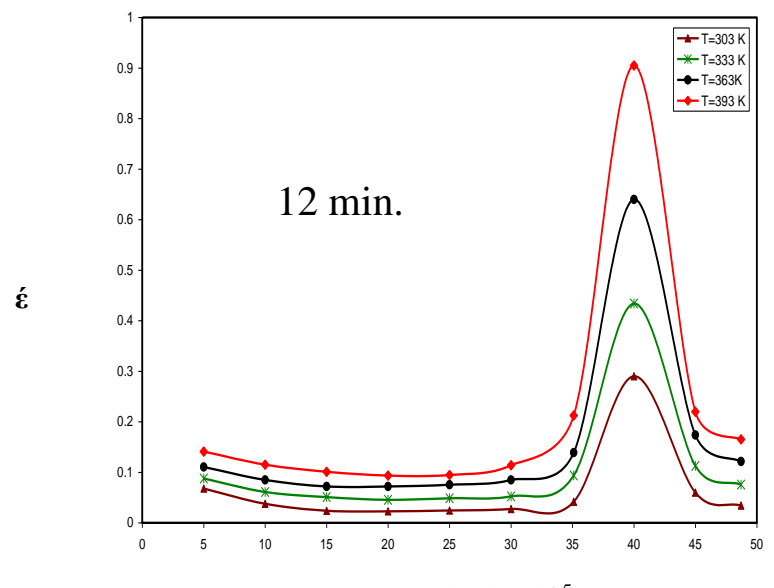

(F)

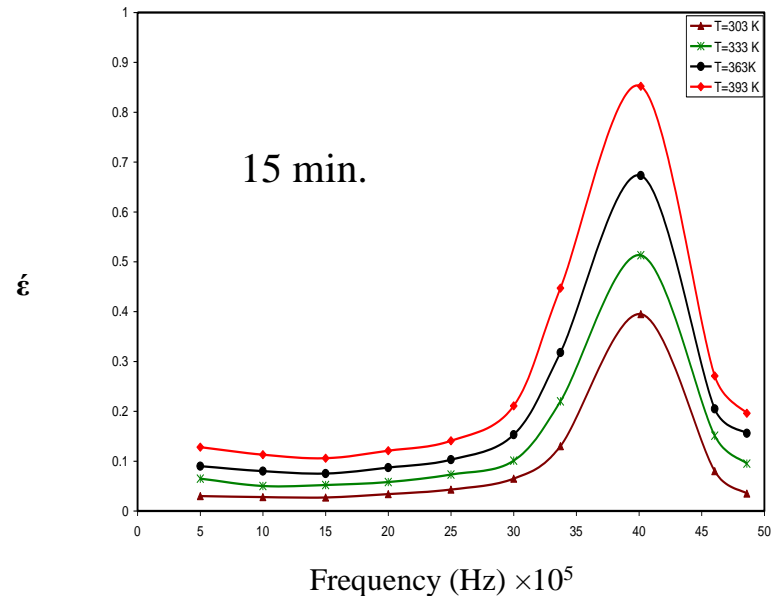

Fig. 3: (A-F)): Variation of Dielectric Constant for PANI/PVA Composite and PANI/PVA Nanocomposite with Irradiation Time at (3, 6, 9, 12 and 15) Min., As Function of Frequency at Different Temperature.

The variation of the real part of dielectric constant for PANI/PVA composite and PANI/PVA nanocomposite films slightly decreased with increasing of frequency up to $2.5 \mathrm{MHz}$. This may be attributed to the tendency of dipoles in PANI/PVA composite and PANI/PVA nanocomposite to orient themselves in the direction of the applied field. However, by increasing frequency the dielectric $(\varepsilon)$ constant increases to reach a maximum value about $4 \mathrm{MHz}$. This may be due to the release of the frozen dipoles and their cooperative motion with adjoining segments in the main chain and this frequency the molecule started to rearrange their conformations. The dielectric values were decreased again in the PANI/PVA composite and PANI/PVA nanocomposite up to $5 \mathrm{MHz}$. This may be due to the increase of charge carriers in which decrease the dielectric constant. The effect of high frequency on $(\varepsilon)$ is very clear in all samples under investigation such that by increasing frequency, $(\dot{\varepsilon})$ It deceases until it reaches a minimum at $5 \mathrm{MHz}$. It could be explained by dipoles orientation, which difficult to rotate at high-frequency range [23]. On the other hand, it can explain the increase of the dielectric permittivity with increasing temperature by the increase in polarization as the segmental

(A)

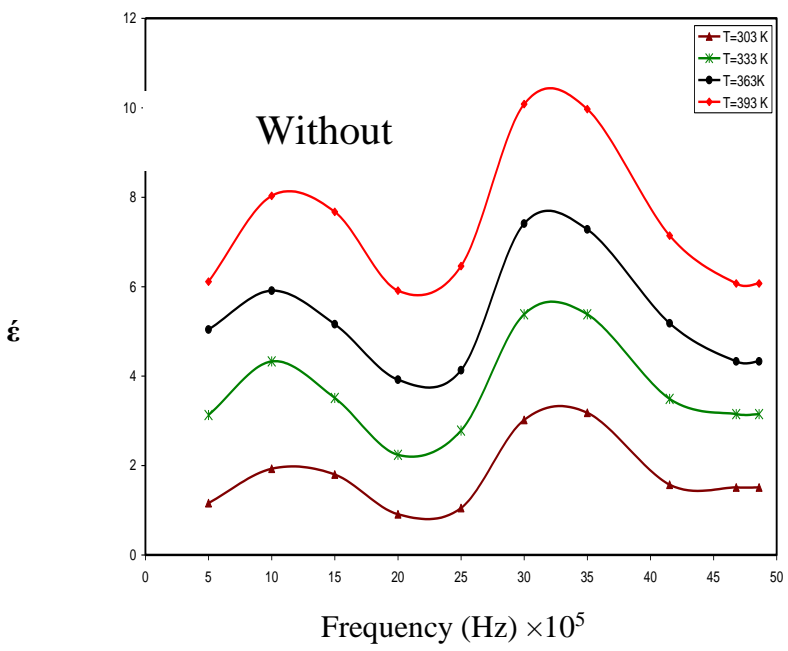

parts are oriented by the external field accompanied by the applied frequency. It can also be seen that, the dielectric constant almost decreases with the increase of frequency due to the dielectric dispersion. This is because the dipoles cannot follow the alternation of the field without a measurable lag because of internal retarding or friction forces.

\subsubsection{Dielectric loss}

The variation of dielectric loss $\varepsilon$ " for PANI/PVA composite and PANI/PVA nanocomposite with increasing the irradiation times, at $(3,6,9,12$ and 15) mints, as a function of frequency in range $(500 \mathrm{KHz}-5 \mathrm{MHz})$ at different temperatures $(303,333,363$ and 393 $\mathrm{K}^{\circ}$ ) is shown in Fig. (4 (a-f)). From these figures, clearly, all dielectrics have two types of losses [24]. One is a conduction loss, representing the flow of actual charge through the dielectric. The other is a dielectric loss due to movement or rotation of the atoms or molecules in an alternating electric field.

(B)

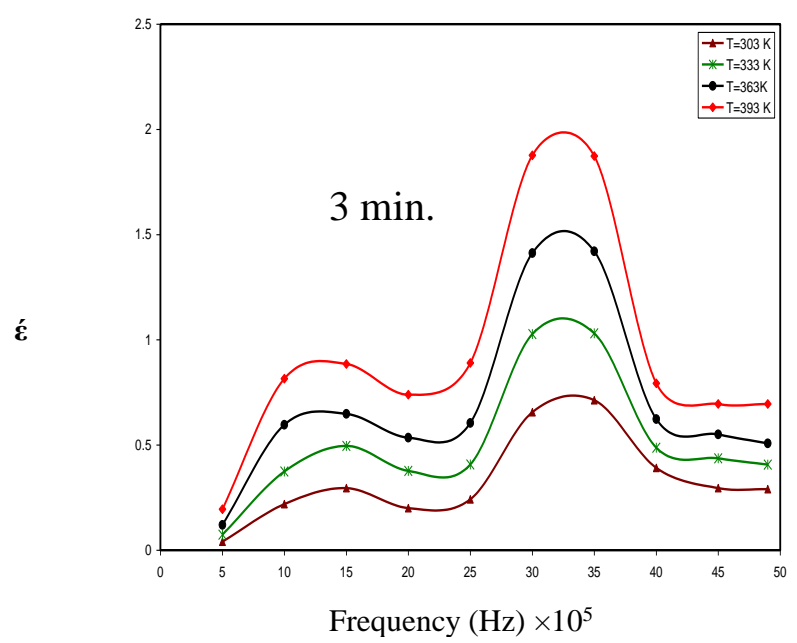


(C)

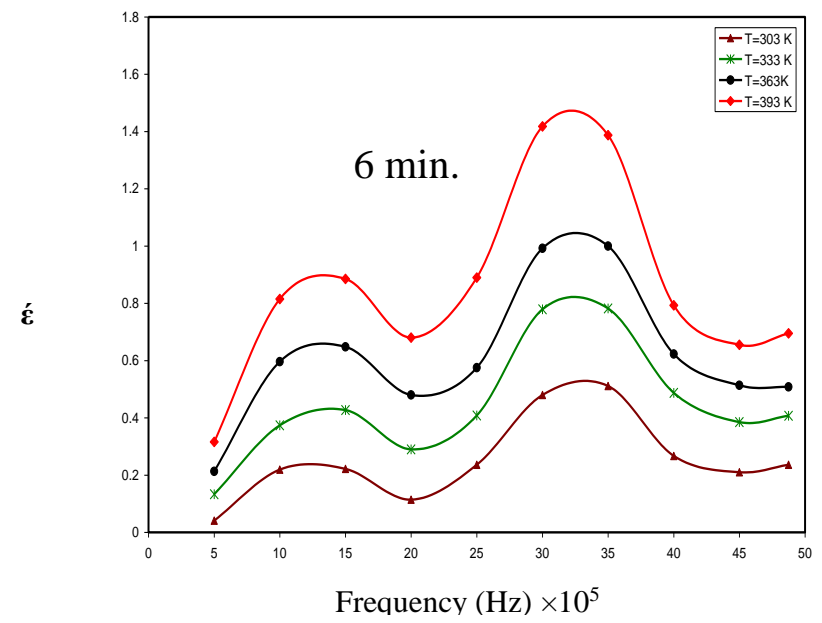

(E)

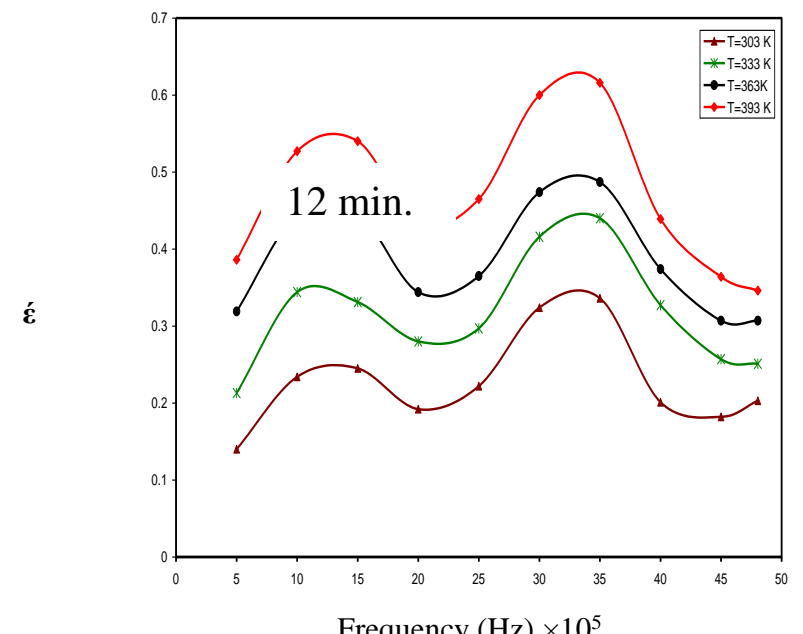

(D)

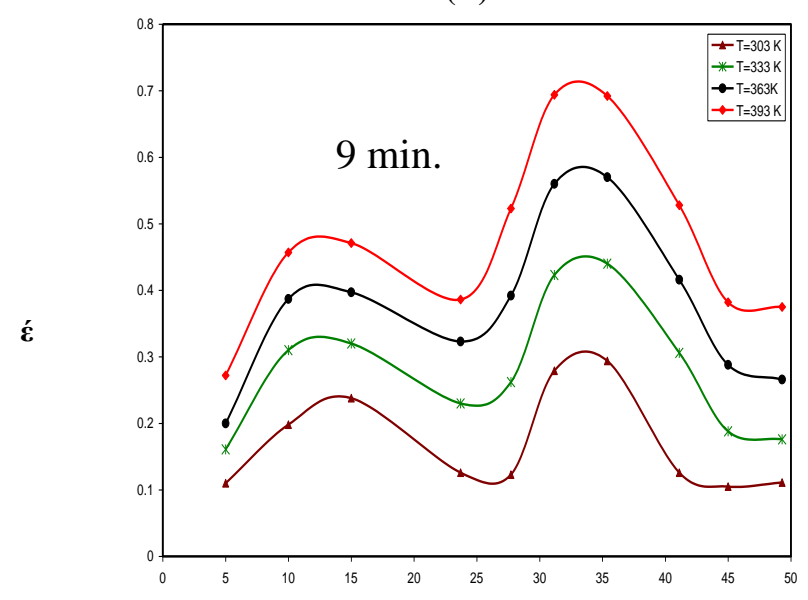

Frequency $(\mathrm{Hz}) \times 10^{5}$

(F)

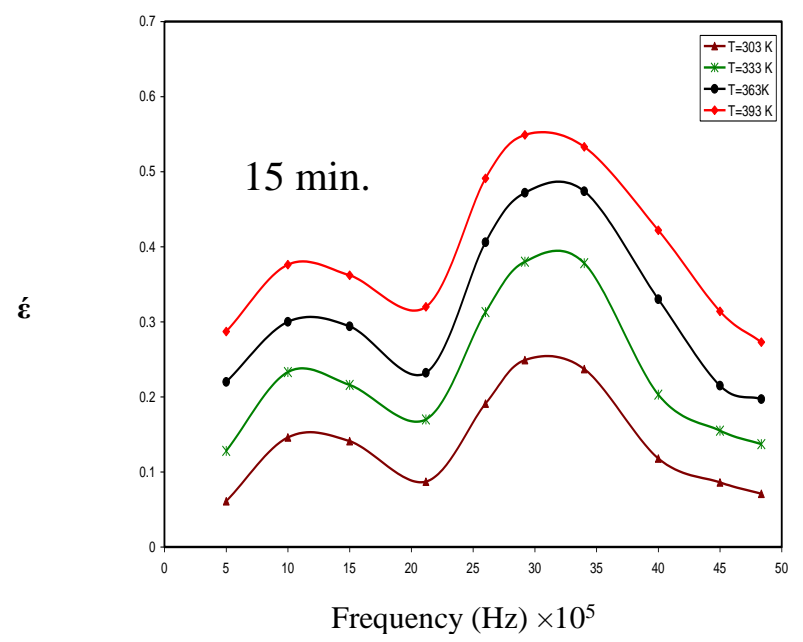

Fig. 4: (A-F)): Variation of Dielectric Loss of PANI/PVA Composite without and with Irradiation Time at (3, 6, 9, 12 and 15) Min. as Function of Frequency at Different Temperatures.

Dielectric loss exhibits two loss maximum; one around lower frequency and the other around higher frequency. The first dielectric loss maximum at lower frequency, $\approx 15 \mathrm{KHz}$ corresponds to the-relaxation peak and the second dielectric loss maximum at higher frequency, $\approx 3.5 \mathrm{KHz}$ depicts $\alpha^{\prime}$-relaxation peak, may be assigned to ion's migration in the disordered structure of the PANI/PVA nanocomposite as indicated by Einfeldt et al .[25], [26].

Also, this is may be attributed to the increased mobility of polar groups and increase in the number of mobile dipoles at this frequency [27]. Also, after the maximum peak at $\approx 3.5 \mathrm{MHz}$ the $(\varepsilon ")$ was decreased again due to the low distributed of the orientational dipole due to the decreasing in the crosslinkage [22].

\subsubsection{Dielectric loss angle (dissipation factor)}

The variation of loss factor $(\mathrm{D}=\tan \delta)$ with frequency at different temperatures of PANI/PVA composite and PANI/PVA nanocomposite with increasing the irradiation times, at (3, 6, 9, 12 and 15) min., as a function of frequency range $(500 \mathrm{kHz}$ to $5 \mathrm{MHz})$ at different temperatures $\left(303,333,363\right.$, and $\left.393 \mathrm{~K}^{\circ}\right)$ are shown in Fig. (5 (a-f)). From these Figures it is clear that $(\tan \delta)$ increases with the increase of temperature at all frequencies. The variation is most prominent at lower frequencies. At higher temperatures the decrease in $(\tan \delta)$ with frequencies is more rapid. The increase in $(\tan \delta)$ especially at higher temperature and in the low frequency region is presumably due to the increase in the number of thermally generated free carriers [28]
Therefore, we determined composites by observing the frequencyindependent value of $\tan \delta$ obtained from a multi frequency plot of $\tan \delta$ vs. frequency. The general trend is a steady decrease in the $\tan \delta=\left(\varepsilon^{\prime \prime} / \varepsilon\right)$ values with increasing frequency due to the relatively dominant contribution of the storage dialectical constant to the loss constant. By comparing ( $\tan \delta$ ) of PANI/PVA composite with PANI/PVA nanocomposite, it can be seen that, the phase transition of PANI/PVA composite at frequency $(27.8) \times 10^{5} \mathrm{~Hz}$, shifted to $(28.09,29.29,30.37,31.79$ and 33.2$) \times 10^{5} \mathrm{~Hz}$, for PANI/PVA nanocomposite at (3,6,9,12 and 15 )min., respectively. 
(A)

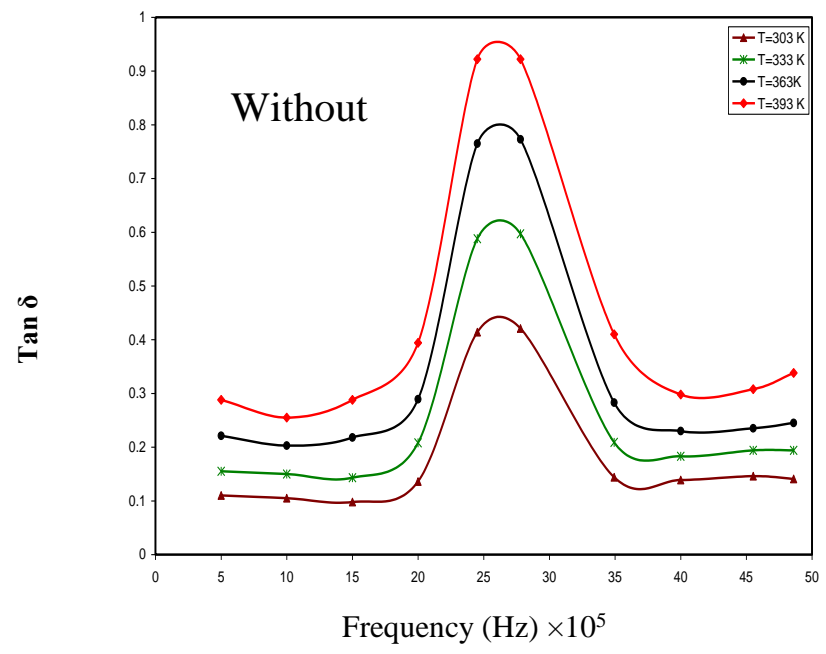

(C)

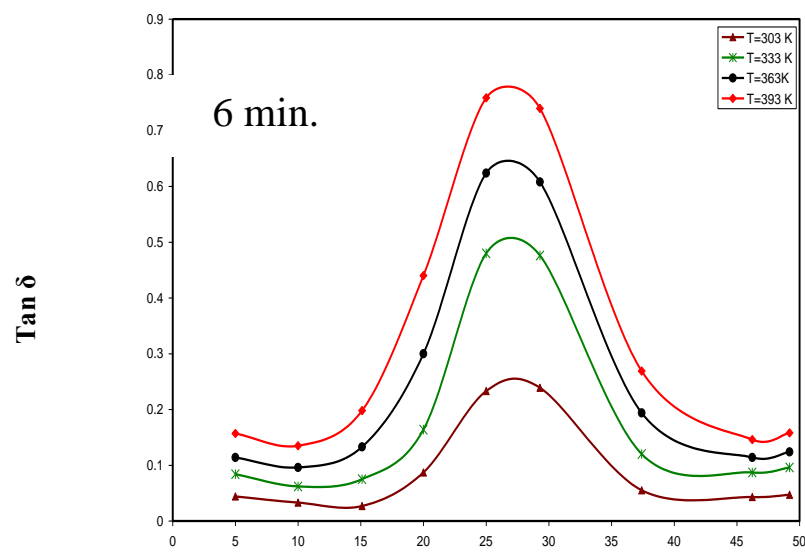

Frequency $(\mathrm{Hz}) \times 10^{5}$

(E)

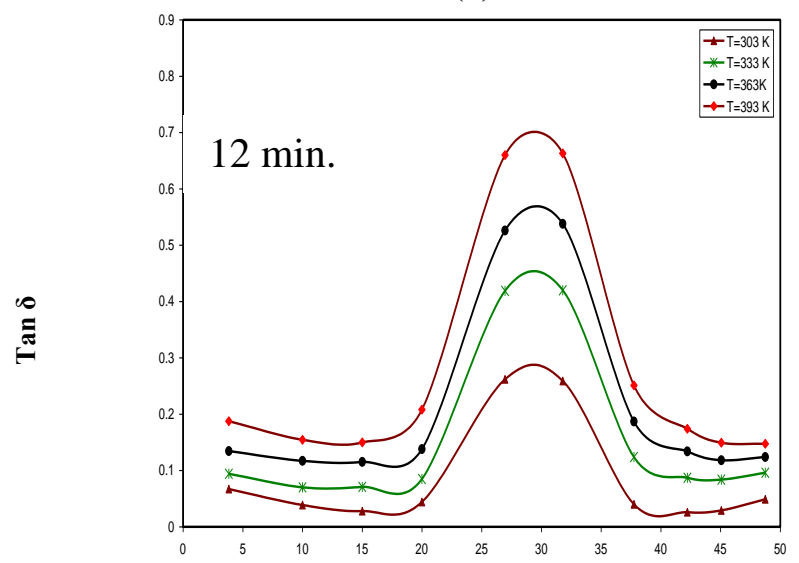

Frequency $(\mathrm{Hz}) \times 10^{5}$
(B)

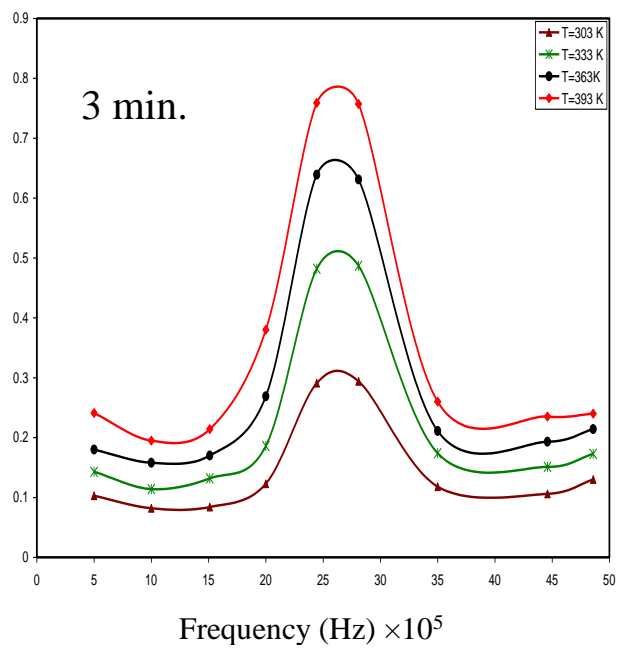

(D)

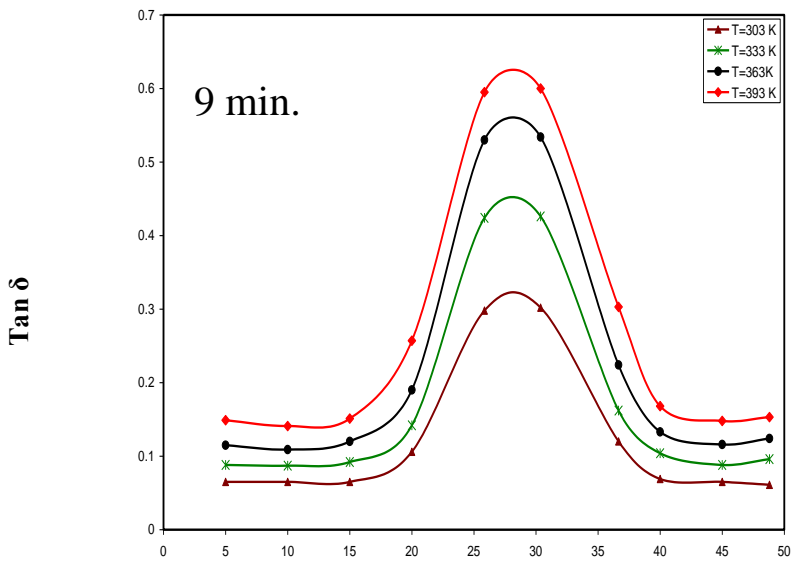

Frequency $(\mathrm{Hz}) \times 10^{5}$

(F)

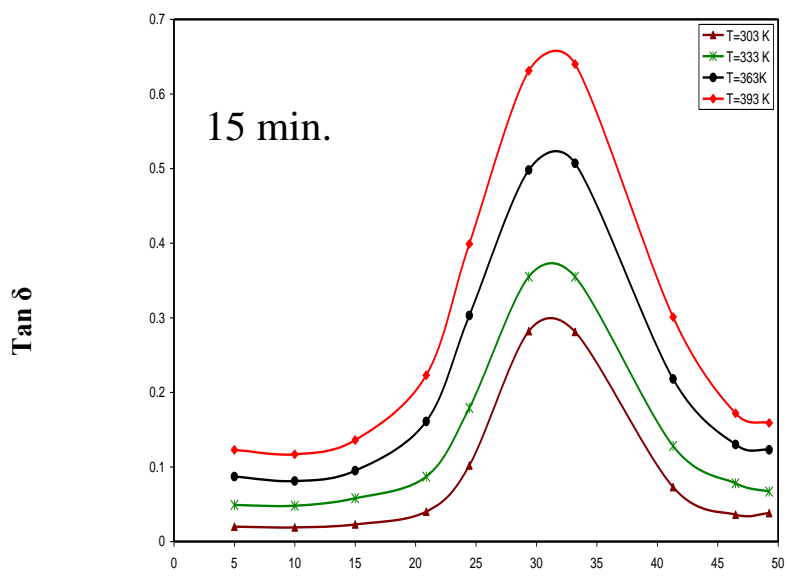

Frequency $(\mathrm{Hz}) \times 10^{5}$

Fig. 5: (A-F)): Variation of Loss Factor of PANI/PVA Nanocomposite without and with Irradiation Time at (3, 6, 9, 12 And 15) Min., as Function of Frequency at Different Temperature.

The apparent relaxation times are calculated from the equation:

$\log (\tau)=\log \left(\frac{\varepsilon^{\prime \prime}}{\omega}\right)-\log \left(\dot{\varepsilon}-\varepsilon_{\infty}\right)$

Where $\omega$ is the angular frequency and, $\varepsilon_{\infty}$ is the unrelaxed constant (the dielectric constant obtained at such a high frequency that no dipole relaxation takes place) and $\tau$ is the relaxation time.
Applying linear fitting for the data in Fig. (6 (a-f)), the values of relaxation time $(\tau)$ could be obtained for the investigated samples with increasing the irradiation times, at (3, 6, 9, 12 and 15) min., as a function of frequency at different temperatures are shown in Figure and are listed in Tables (2). From this tables, it can be observed that relaxation time of PANI/PVA composite is higher than that of PANI/PVA nanocomposite due to stronger bonds in pure polyaniline chain than nanocomposite. This attributed to the molecular motion in this transition and the dipoles are more free in 
the present sample.This can be attributed to the incorporation of ions between the polymer chains which make destruction in the internal interaction. Also it can be observed that the relaxation times become shorter as the irradiation increased which indicates that multiple path of the system to be relaxed due to high availability of free charges. From the whole data, it can conclude that the relaxation time is relatively reduced with increasing temperature of sample; this may be due to intrinsic behavior [24].

Table 2: Relaxation Time for PANI/PVA Composite before and after Different Irradiation Time

\begin{tabular}{lccll}
\hline & \multicolumn{2}{l}{ PANI/PVA composite } & \multicolumn{2}{l}{$\begin{array}{l}\text { PANI/PVA composite at } \\
\text { Pin. }\end{array}$} \\
\hline $\begin{array}{l}\text { Temperature } \\
(\mathrm{K})\end{array}$ & $\log (\tau)$ & $\tau$ & $\log (\tau)$ & $\tau$ \\
303 & -7.509 & $5.5 \mathrm{x}^{-7}$ & -7.512 & $5.5 \mathrm{x}^{-7}$ \\
333 & -7.404 & $5.8 \mathrm{x}^{-7}$ & -7.454 & $5.7 \mathrm{x}^{-7}$ \\
363 & -7.326 & $6.4 \mathrm{x}^{-7}$ & -7.436 & $5.8 \mathrm{x}^{-7}$ \\
393 & -7.303 & $6.7 \mathrm{x}^{-7}$ & -7.423 & $5.9 \mathrm{x}^{-7}$ \\
\hline
\end{tabular}

\begin{tabular}{|c|c|c|c|c|}
\hline & \multicolumn{2}{|c|}{$\begin{array}{l}\text { PANI/PVA composite at } 6 \\
\text { min. }\end{array}$} & \multicolumn{2}{|c|}{$\begin{array}{l}\text { PANI/PVA composite at } \\
9 \mathrm{~min} \text {. }\end{array}$} \\
\hline $\begin{array}{l}\text { Temperature } \\
\text { (K) }\end{array}$ & $\log (\tau)$ & $\tau$ & $\log (\tau)$ & $\tau$ \\
\hline 303 & -7.510 & $5.5 \mathrm{x}^{-7}$ & -.5303 & $5.3 \mathrm{x}^{-7}$ \\
\hline 333 & -7.488 & $5.59 \mathrm{x}^{-7}$ & -7.524 & $5.4 \mathrm{x}^{-7}$ \\
\hline 363 & -7.477 & $5.6 x^{-7}$ & -.5158 & $6.4 \mathrm{x}^{-7}$ \\
\hline \multirow[t]{2}{*}{393} & -7.469 & $5.7 x^{-7}$ & -.5095 & $6.7 x^{-7}$ \\
\hline & \multicolumn{2}{|c|}{$\begin{array}{l}\text { PANI/PVA composite at } \\
12 \mathrm{~min} .\end{array}$} & \multicolumn{2}{|c|}{$\begin{array}{l}\text { PANI/PVA composite at } \\
15 \mathrm{~min} \text {. }\end{array}$} \\
\hline $\begin{array}{l}\text { Temperature } \\
\text { (K) }\end{array}$ & $\log (\tau)$ & $\tau$ & $\log (\tau)$ & $\tau$ \\
\hline 303 & -7.569 & $5.1 \mathrm{x}^{-7}$ & -7.722 & $5.4 x^{-7}$ \\
\hline 333 & -7.550 & $5.2 \mathrm{x}^{-7}$ & -7.664 & $5.7 x^{-7}$ \\
\hline 363 & -7.544 & $5.31 \mathrm{x}^{-7}$ & -7.523 & $5.8 x^{-7}$ \\
\hline 393 & -7.537 & $5.37 \times-7$ & -.5713 & $5.9 x^{-7}$ \\
\hline
\end{tabular}
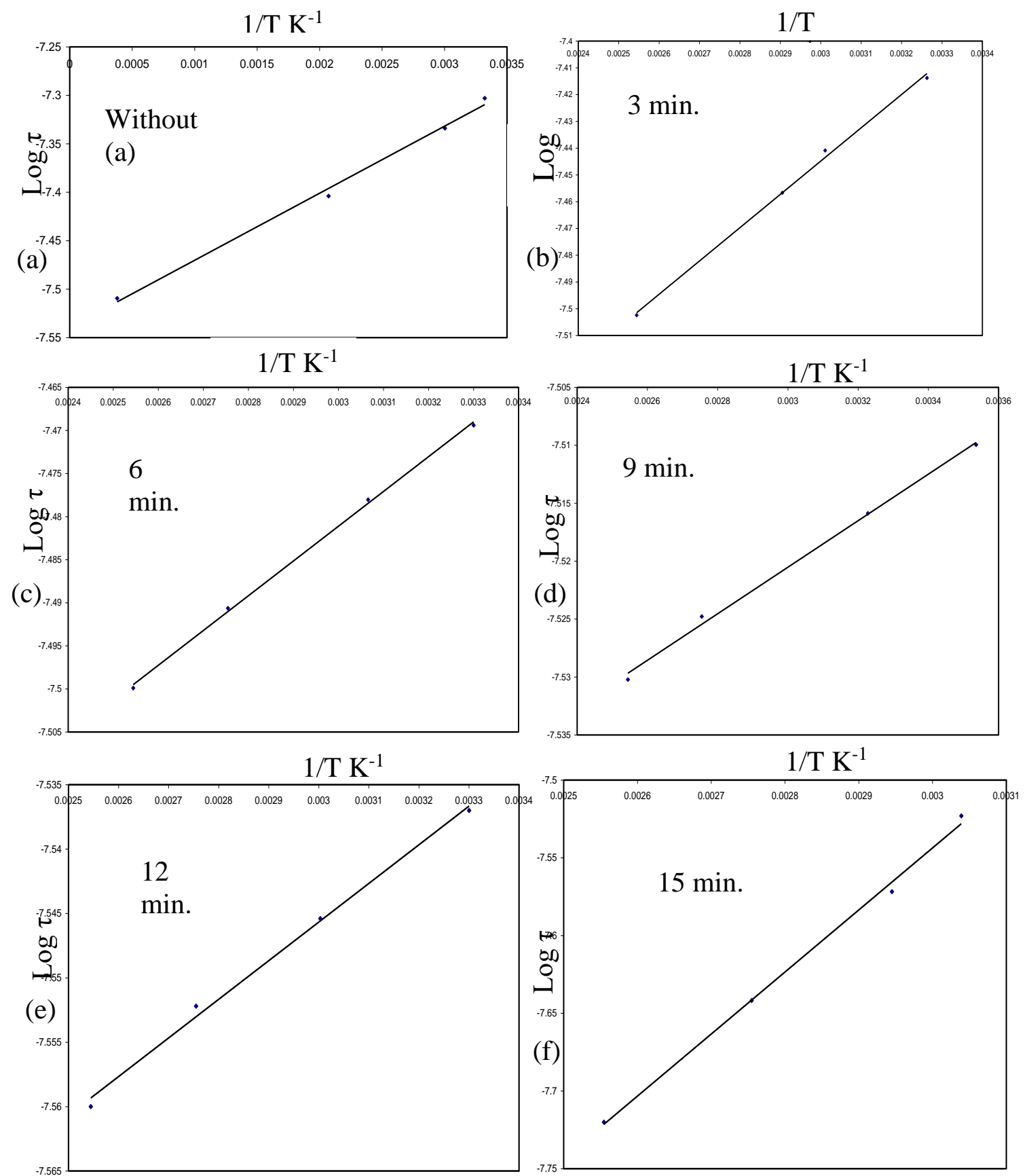

Fig. 6: (A-F): the Relation between $\log \mathrm{T}$ and $1 / \mathrm{T}(\mathrm{K})^{-1}$ for PANI/PVA Nanocomposite before and after Irradiation Times At $(3,6,9,12$ and 15$)$ Min., as Function of Frequency at Different Temperature. 


\section{Conclusions}

XRD pattern of PANI/PVA nanocomposite suggests that, it has an amorphous structure, and the particle size decrease with increasing irradiation times (3 6, 9, 12 and 15) min. The electrical properties have been determined and discussed.

\section{References}

[1] J S Shin, J H Kima, I W Cheong (Colloidal and thermal stability of polyaniline-coated multi-core shell polystyrene latexes prepared using sulfonated N-hydroxyethyl aniline) Synthetic Metals 151246 (2005). https://doi.org/10.1016/j.synthmet.2005.05.006

[2] T Thanpitcha, A Sirivat, A M Jamieson , R Rujiravanit (Preparation and characterization of polyaniline/chitosan blend film)Carbohydrate $\quad$ Polymers $\quad 64 \quad 560 \quad$ (2006) https://doi.org/10.1016/j.carbpol.2005.11.026.

[3] Z Wang, J Chen, X Xue, Y Hu (Synthesis of monodispersed CdS nanoballs through $\gamma$-irradiation route and building core-shell structure CdS@SiO2) Mater. Res. Bull. 42 2211(2007). https://doi.org/10.1016/j.materresbull.2007.01.002.

[4] S Wang, G Shi, (Uniform silver/polypyrrole core-shell nanoparticles synthesized by hydrothermal reaction) Mater. Chem. Phys. 102 255 (2007). https://doi.org/10.1016/j.matchemphys.2006.12.014.

[5] C Song, D. Wang, Y. Lin, Z.Hu, G. Gu, \& X. Fu (Formation of silver nanoshells on latex spheres) Nanotech. 15962 (2004). https://doi.org/10.1088/0957-4484/15/8/016.

[6] F Caruso, (Nanoengineering of Particle Surfaces) Adv. Mater. 13 11 (2001). https://doi.org/10.1002/1521 4095(200101)13:1<11::AID-ADMA11>3.0.CO;2-N

[7] A V B reddy, TJR Sharma, A K NarasimhaRao (Structural, Electrical and Optical Characterization of Pure and Doped Poly (Vinyl Alcohol) (PVA) Polymer Electrolyte Films) Int.J.of Polymeric Mater 56579 (2007).

[8] P Scherrer, (The Scherrer Formula for X-Ray Particle Size Determination) G Nachrichten 298 (2011).

[9] S Bhadra, N K Singha, and D Khastgir (Dual functionality of PTSA as electrolyte and dopant in the electrochemical synthesis of polyaniline, and its effect on electrical properties) Polym, Int., 56919 (2007). https://doi.org/10.1002/pi.2225.

[10] F Zuo, et al. (Solution Studies of the Emeraldine Oxidation State of Polyaniline) Synthetic Metals, $29 \quad 445 \quad$ (1996). https://doi.org/10.1016/0379-6779(89)90331-7.

[11] S Zhang, S Kan, and J Kan (Chemical synthesis and characterization of polyaniline nanofiber doped with gadolinium chloride) Journal of Applied Polymer Science, 100946 (2006). https://doi.org/10.1002/app.23072.

[12] P P Sengupta, and B Adhikari (Influence of polymerization condition on the electrical conductivity and gas sensing properties of polyaniline) Materials Science and Engineering: A., 459278 (2007). https://doi.org/10.1016/j.msea.2007.02.021.

[13] R Cruz-Silva, (Ph-and thermosensitivepolyaniline colloidal particales prepared by enzymatic polymerization) Langmuir 238 (2007). https://doi.org/10.1021/la0618418.

[14] W Yin and E Ruckenstein (Soluble polyaniline co-doped with dodecyl benzene sulfonic acid and hydrochloric acid) Synth. Met., 10839 (2000). https://doi.org/10.1016/S0379-6779(99)00179-4.

[15] B C Roy, M D Gupta, L Bhoumik and J K Ray (Synthesis and characterization of conducting poly (1- aminonaphthalene), poly (2aminonaphthalene) and poly (aniline-co-1aminonaphthalene).Synth. $\quad$ Met., $\quad 130 \quad 27 \quad$ (2002). https://doi.org/10.1016/S0379-6779(02)00108-X.

[16] M Kumar, O Yavuz, V Lahsangah, M Aldissi (CO gas sensing from ultrathin nano-composite conducting polymer film) Sensors and

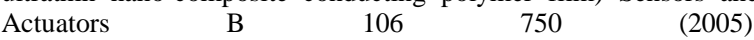
https://doi.org/10.1016/j.snb.2004.09.027.

[17] A G MacDiarmid and A J Epstein (The Concept of Secondary Doping as Applied to Poly aniline) Synth. Met., 65103 (1994). https://doi.org/10.1016/0379-6779(94)90171-6.

[18] B G Choi, H Park, H S Im, Y J Kim, and W H Hong (Influence of oxidation state of polyaniline on physicochemical and transport properties of nafion /polyaniline composite membrane of DMFC.) Journal of Membrane Science $324 \quad 102 \quad$ (2008). https://doi.org/10.1016/j.memsci.2008.06.061.

[19] S Xing, H Zheng and G Zhao (Preparation of Polyaniline Nanofibers via a Novel Interfacial Polymerization Method) Synthetic
Metals 158 59 https://doi.org/10.1016/j.synthmet.2007.12.004.

[20] O Ngamna et al. (Inkjet printable polyaniline nanoformulations) Langmuir 238569 (2007). https://doi.org/10.1021/la700540g.

[21] N E Hill, W E Vaughen, H Price and M Davis "Dielectric Properties and Molecular Behaviour" (London: Van Nostrand, 2004).

[22] A Elshafie, H H Afify and A Bbdel All "Dielectric properties of Tungsten oxide thin film prepared by spray pyrolysis" Phys. Stat. Sol. $174301 \quad$ (1999). https://doi.org/10.1002/(SICI)1521396X(199907)174:1<301::AID-PSSA301>3.0.CO;2-G

[23] P Dutta, S Biswas and S De, "Dielectric relaxation in polyaniline/polyvinyle-alchohol composite" Materials Research Bulletin 37 193 (2002). https://doi.org/10.1016/S0025-5408(01)00813-3.

[24] W Sarjeant, James and R E Dollinger High-Power Electronics, TAB Books (2006).

[25] Einfeldt, D Meißner and A Kwasniewski, "Contributions to the molecular origin of the dielectric relaxation process in polysaccharides-the higher temperature range" J.Non-Crys. Solids 32040 (2003).

[26] J Einfeldt, D Meißner and A Kwasniewski, "Polymerdynamics of cellulose and other polysaccharides in solid state-secondary dielectric relaxation processes", Prog. Polym. Sci. 26 1419(2001) https://doi.org/10.1016/S0079-6700(01)00020-X.

[27] M H Harun et al."Dielectric properties of poly (vinyl alchohol)/polypyrrole composite polymer films" J. Advanc. Scien. Arts 1 9 (2009).

[28] K P Singh and P N Gupta."Study of dielectric relaxation in polymer electrolytes" European Polymer Journal $34 \quad 1023 \quad$ (1998). https://doi.org/10.1016/S0014-3057(97)00207-3. 